\title{
Knockdown of phosphoinositide-dependent kinase 1 (PDK1) inhibits fibrosis and inflammation in lipopolysaccharide-induced acute lung injury rat model by attenuating NF-kB/p65 pathway activation
}

\author{
Keke Yang, Boqian Li, Jinghua Chen \\ Jiamusi College, Heilongjiang University of Traditional Chinese Medicine, Jiamusi, China \\ Contributions: (I) Conception and design: K Yang, J Chen; (II) Administrative support: B Li; (III) Provision of study materials or patients: K Yang, B \\ Li; (IV) Collection and assembly of data: K Yang, B Li; (V) Data analysis and interpretation: K Yang, J Chen; (VI) Manuscript writing: All authors; \\ (VII) Final approval of manuscript: All authors. \\ Correspondence to: Jinghua Chen. Jiamusi College, Heilongjiang University of Traditional Chinese Medicine, 53 Guanghua Street, Jiamusi 154007, \\ China. Email: chenjinghua234678@163.com.
}

Background: Acute lung injury (ALI) is a common inflammatory disease of the lung. This study aimed to investigate the effect of 3-phosphoinositide-dependent kinase 1 (PDK1) interference on the levels of fibrosis and proinflammatory factors in lipopolysaccharide (LPS)-induced ALI and discuss the relevant mechanism.

Methods: An ALI model was established by intravenous injection of LPS treatment. A total of 24 SpragueDawley (SD) rats were randomly divided into 4 groups: sham group; ALI group; ALI + shRNA-NC group; and ALI + PDK1-shRNA group. Lung injury score, minute ventilation, lung volume, and airway resistance were used to evaluate lung function injury. Reverse transcription-polymerase chain reaction (RTPCR) was used to detect PDK1 messenger RNA (mRNA) level. Western blot was performed to detect expression levels of PDK1, transforming growth factor- $\beta$ (TGF- $\beta$ ), $\alpha$-smooth muscle actin ( $\alpha$-SMA), toll-like receptor 4 (TLR4), p65, and myeloid differentiation primary response gene 88 (MyD88). The contents of interleukin-6 (IL-6), inducible nitric oxide synthase (iNOS), tumor necrosis factor- $\alpha$ (TNF- $\alpha$ ), and monocyte chemoattractant protein-1 (MCP-1) were detected by enzyme-linked immunosorbent assay (ELISA). The pathological changes and fibrosis of lung tissues were estimated by hematoxylin and eosin (H\&E) and Masson staining.

Results: The results revealed that high lung injury score, low minute ventilation, low lung volume, and small airway resistance were present in the ALI group. Likewise, severe histopathological damage and fibrosis were apparent in the ALI group. Otherwise, contents of TNF- $\alpha$, iNOS, IL-6, MCP-1, and levels of $\alpha$-SMA, TGF- $\beta$, TLR4, phosphorylated (p)-p65, and MyD88 were enhanced in the ALI group. Interestingly, pathological changes and fibrosis were improved significantly in the ALI + PDK1-shRNA group. Besides, knockdown of PDK1 reduced lung injury score and enhanced minute ventilation, lung volume, and airway resistance. Moreover, knockdown of PDK1 decreased the contents of TNF- $\alpha$, iNOS, IL-6, MCP-1, and levels of TGF- $\beta, \alpha$-SMA, TLR4, p-p65, and MyD88.

Conclusions: Knockdown of PDK1 protects LPS-induced ALI via attenuating activation of the nuclear factor- $\kappa \mathrm{B}(\mathrm{NF}-\kappa \mathrm{B}) / \mathrm{p} 65$ pathway.

Keywords: Acute lung injury (ALI); protein kinase; phosphoinositide-dependent kinase 1 (PDK1); nuclear factor$\kappa \mathrm{B}(\mathrm{NF}-\kappa \mathrm{B}) / \mathrm{p} 65$

Submitted Sep 13, 2021. Accepted for publication Nov 09, 2021.

doi: $10.21037 / \mathrm{atm}-21-5476$

View this article at: https://dx.doi.org/10.21037/atm-21-5476 


\section{Introduction}

Acute lung injury (ALI) is a common inflammatory disease of the lung that causes hypoxemia, edema, and respiratory failure (1). The histological characteristics of ALI are hemorrhage, diffuse inflammatory alveolar infiltration, hyaline membrane formation, and pulmonary edema $(2,3)$. The results of epidemiologic studies have suggested that ALI is still a challenge for clinicians and the global public health system (4). Although numerous therapeutic methods have been adopted to ameliorate the functional prognosis, the mortality of ALI is still very high $(5,6)$. Thus, it is necessary to confirm the pathogenesis of ALI and explore new therapeutic strategies.

Lipopolysaccharide (LPS), the main component of the outer membranes of gram-negative bacteria, is a proven inducer of ALI (7). In this process, LPS activates neutrophils and nicotinamide adenine dinucleotide phosphate (NADPH) oxidase to generate oxygen free radicals, which eventually leads to ALI (8). Hence, understanding the underlying mechanism of LPS-induced inflammatory injury is crucial for the development of new therapeutic strategies for ALI $(9,10)$. Induction of LPS has been widely considered an effective strategy to establish ALI animal models by inducing inflammation $(11,12)$.

The 3-phosphoinositide dependent protein kinase-1 (PDK1) is a major regulatory protein kinase and a member of A, G, C (AGC) protein kinase families (13). It has been found that PDK1 is an upstream activator, which can activate multiple downstream effectors and can be involved in the development of a variety of diseases and cancer (14). Studies have shown that PDK1 activity is closely related to the occurrence and development of inflammatory responses, and inhibition of PDK1 can suppress systemic inflammation (15).

Nuclear factor- $\kappa \mathrm{B}(\mathrm{NF}-\kappa \mathrm{B})$ is a critical transcription factor located downstream of the Toll-like receptor signaling pathway. Activated $\mathrm{I} \kappa \mathrm{B} \alpha$ is phosphorylated and degraded, causing NF- $\mathrm{\kappa B} / \mathrm{p} 65$ displacement to the nucleus to bind inflammation-related genes, thereby stimulating the release of pro-inflammatory mediators (16). A series of studies have shown that NF- $\kappa \mathrm{B}$ is involved in LPSinduced ALI $(17,18)$. During the process, NF- $\kappa B$ activation is mainly related to the production of inflammatory factors such as tumor necrosis factor- $\alpha$ (TNF- $\alpha$ ), interleukin (IL)-6, and IL-1 $\beta(18,19)$.

In the present study, we discussed the effects of PDK1 inhibition on the levels of fibrosis and inflammation in an
LPS-induced ALI rat model. In addition, the relationship

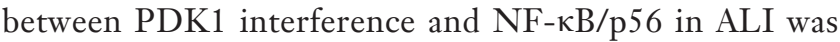
discussed. We present the following article in accordance with the ARRIVE reporting checklist (available at https:// dx.doi.org/10.21037/atm-21-5476).

\section{Methods}

\section{Reagents and rats}

We purchased LPS from Sigma-Aldrich (Escherichia coli 055:B5; Sigma-Aldrich, St. Louis, MO, USA); short hairpin RNA (shRNA)-NC and PDK1-shRNA were produced by RiboBiotech (Ribo-Bio, Guangzhou, China). Additionally, we purchased Weigert's iron hematoxylin working solution (solution A), Biebrich scarlet-acid fuchsin solution (solution B), phosphomolybdic-phosphotungstic acid solution (solution C), aniline blue solution (solution D), and acetic acid solution (solution E) from Xinbosheng Biotechnology Co., Ltd. (Shenzhen, China). The enzymelinked immunosorbent assay (ELISA) kit was obtained from eBioscience (San Diego, CA, USA). Sprague-Dawley (SD) rats (6-8 weeks, 250-300 g) were obtained from the Animal Experiment Center of Sichuan University (Chengdu, China), and were allowed free access to food and water.

\section{Animal experiments}

The ALI was induced by LPS treatment as previously described (20). Rats were randomly allocated to 4 groups (with 6 in each group): sham group: intratracheally instilled with $3 \mathrm{mg} / \mathrm{kg}$ normal saline; ALI group: intratracheally instilled with $3 \mathrm{mg} / \mathrm{kg}$ LPS; ALI + shRNANC group: intratracheally instilled with $3 \mathrm{mg} / \mathrm{kg}$ LPS + $8 \mathrm{mg} / \mathrm{kg}$ shRNA-NC; and ALI + PDK1-shRNA group: intratracheally instilled with $3 \mathrm{mg} / \mathrm{kg}$ LPS $+8 \mathrm{mg} / \mathrm{kg}$ PDK1-shRNA (pre-treatment dose). After $48 \mathrm{~h}$, animals were anesthetized by intraperitoneal injection of pentobarbital sodium $(50 \mathrm{mg} / \mathrm{kg})$ and euthanized by bloodletting. Bronchoalveolar lavage (BAL) fluid was collected to detect the levels of inflammatory cytokines. Lung tissue was separately harvested and stored at $-80{ }^{\circ} \mathrm{C}$ for the following experiments. Serum samples were collected from the main artery of each rat and plasma was isolated by centrifugation $(12,000 \times \mathrm{g}, 12 \mathrm{~min})$ and stored at $-80^{\circ} \mathrm{C}$

Animal experiments were conducted according to the declaration of the Guide for the Care and Use of Laboratory 
Animals (2011) and approved by the ethics committee of Heilongjiang University of traditional Chinese Medicine (No. 2020136). A protocol was prepared before the study without registration.

\section{Reverse transcription-polymerase chain reaction (RT-PCR)}

Before RT, Total RNA of lung tissues was extracted by TRIzol reagent (Invitrogen, Carlsbad, CA, USA) to prepare complementary DNA (cDNA; PrimeScript RT reagent Kit with gDNA Eraser; Takara Bio., Kusatsu, Shiga, Japan). The real-time PCR analysis of cDNA was operated by ABI Q3 and a SYBR Prime Script RT Reagent Kit (Takara). All quantitative analyses were made in triplicate and standardized as $\beta$-actin. The $2^{-\Delta \Delta c t}$ method was utilized to analyze the relative expression.

PDK1 sequence: forward 5'-TCAACGACCACTTTGTCAAG CTCAGCT-3', reverse 5'-GGTGGTCCAGGGGTCTTAC-3';

$\beta$-actin sequence: forward 5'-CGTCATACTCCTGCTTGCTG-3', reverse 5'-GTACGCCAACACAGTGCTG-3'.

\section{Lung function test}

Lung function injury indexes were estimated according to the previous report (21). The indexes included lung injury score, minute ventilation $(\mathrm{mL} / \mathrm{kg})$, lung volume $(\mathrm{mL})$ and airway resistance. The score of lung injury (including hemorrhage, inflammation, and edema) was graded on the following scale: $0=$ normal, $1=$ light, $2=$ moderate, $3=$ strong, and $4=$ severe. Scoring was performed by pathologists who were blinded to the experiment. The final score was taken as the average of each group.

\section{Histopathology}

Hematoxylin and eosin (H\&E): lung tissues were fixed in $4 \%(\mathrm{v} / \mathrm{v})$ paraformaldehyde and embedded in paraffin. The embedded tissues were cut into $4 \mu \mathrm{m}$ thicknesses and stained with H\&E solution (Sigma-Aldrich, USA). Finally, the sections were observed using a microscope.

Masson: Sections were created as in the H\&E staining process. Then, the sections were stained in solution A for $10 \mathrm{~min}$ and washed with distilled water, followed by staining in solution B for 10 min and washing with distilled water. Next, they were differentiated in solution $\mathrm{C}$ for $10 \mathrm{~min}$ and placed into solution $\mathrm{D}$ for $5 \mathrm{~min}$. After rinsing in distilled water, the sections were differentiated in $1 \%$ solution $\mathrm{E}$ for $2 \mathrm{~min}$ and washed with distilled water. After dehydrating in $95 \%$ and absolute ethyl alcohol, the sections were cleared in xylene. Ultimately, the sections were mounted with resinous mounting medium for observation.

\section{ELISA}

Levels of inducible nitric oxide synthase (iNOS), IL-6, monocyte chemoattractant protein-1 (MCP-1), and TNF- $\alpha$ in BAL were detected by ELISA. The operation processes were conducted according to the manufacturer's instructions.

\section{Western blot analysis}

Total protein $(20 \mu \mathrm{g})$ was extracted from lung homogenates and detected with bicinchoninic acid (BCA) kit. According to the molecular weight of target protein, the total protein in each sample was loaded into 6-12\% sodium dodecyl sulfate-polyacrylamide gel electrophoresis (SDS-PAGE) and then transferred to polyvinylidene difluoride (PVDF) membranes. Next, the membranes were blocked with $5 \%$ skimmed milk and incubated with the primary antibodies overnight at $4{ }^{\circ} \mathrm{C}$. The next day, membranes were washed with Tris-buffered saline with Tween 20 (TBST) 3 times and then incubated with goat anti rabbit IgG horseradish peroxidase (HRP) secondary antibodies for $1 \mathrm{~h}$ at room temperature, followed by washing with TBST 3 times. Antibodies of the following proteins were used: antitransforming growth factor- $\beta$ (anti-TGF- $\beta$ ), anti- $\alpha$-smooth muscle actin (anti- $\alpha$-SMA), anti-IL-6, anti-iNOS, anti-tolllike receptor 4 (anti-TLR4), anti-myeloid differentiation primary response gene 88 (anti-MyD88), anti-p65, antiphosphorylated-p65 (p-p65), and anti-PDK1. The above antibodies were all purchased from Cell Signaling Technology (CST; Danvers, MA, USA).

\section{Statistical analysis}

Analyses were performed using SPSS 22.0 (IBM Corp., Chicago, IL, USA). Analysis of variance (ANOVA) or Students $t$-tests were performed to assess statistical significance, as indicated. Data were presented as the mean \pm standard deviation $(\mathrm{SD})$.

\section{Results}

\section{Construction of PDK1 knockdown model}

As shown in Figure 1A,1B, PDK1 expression level changed with LPS treatment time in rats. The PDK1 expression level reached the maximum at $6 \mathrm{~h}$. To investigate the effect of PDK1 silence on LPS-induced rats, RT-PCR and 

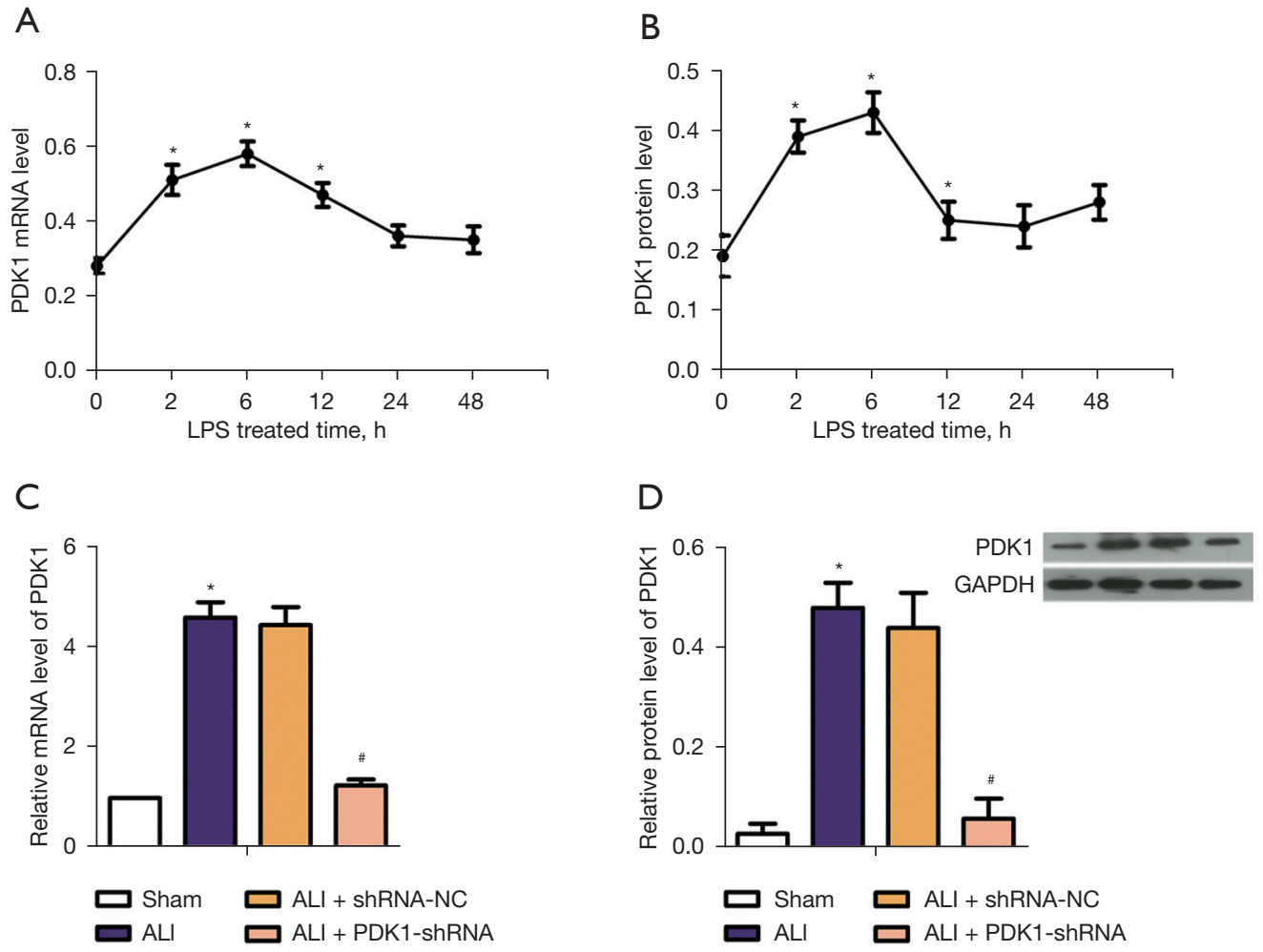

Figure 1 PDK1 expression in rats of each group. (A) The effect of LPS treatment time on the relative mRNA level of PDK1; (B) the effect of LPS treatment time on the relative protein level of PDK1; (C) relative mRNA level of PDK1 in 4 groups; (D) relative protein level of PDK1 in 4 groups. *, $\mathrm{P}<0.05$ vs. the sham group; *, $\mathrm{P}<0.05$ vs. ALI model group. PDK1, 3-phosphoinositide-dependent kinase 1; LPS, lipopolysaccharide; mRNA, messenger RNA; ALI, acute lung injury.

western blot (WB) assay were respectively used to detect messenger RNA (mRNA) level of PDK1 and protein level of PDK1 in vivo. From Figure $1 C, 1 D$, compared with the sham group, the mRNA level and protein level of PDK1 were significantly enhanced in ALI model rats, while PDK1 knockdown significantly decreased these trends. These results demonstrated that the ALI model group and PDK1 silence group had been successfully established.

\section{Effect of PDK1 knockdown on lung function of rats in each group}

To investigate the effect of PDK1 knockdown on pulmonary function of rats, the level of lung injury score, minute ventilation $(\mathrm{mL} / \mathrm{kg})$, lung volume $(\mathrm{mL})$ and airway resistance were detected. As shown in Figure $2 \mathrm{~A}$, lung injury score increased significantly in ALI model rats compared with the sham group; fortunately, this adverse effect was reversed in the ALI + PDK1-shRNA group. As shown in Figure 2B-2D, minute ventilation, lung volume, and airway resistance decreased significantly in ALI model rats compared with the sham group, while knockdown of PDK1 significantly reversed these adverse effects. These data suggested that knockdown of PDK1 could avoid lung function damage to a certain extent.

\section{Effect of PDK1 knockdown on lung pathological damage and inflammatory response}

Pathological damage of the lung was determined by $H \& E$ staining. The images of $H \& E$ staining revealed the microscopic appearance of the lung in each group. As shown in Figure $3 A$, the sham group displayed normal alveoli with intact tissue architecture. Alveolar damage with lymphocyte infiltration was shown in LPS-induced model group, along with marked chronic inflammation and fibroblast proliferation. However, PDK1 knockdown relieved these abnormal phenomena. Myeloperoxidase (MPO) is the 
A

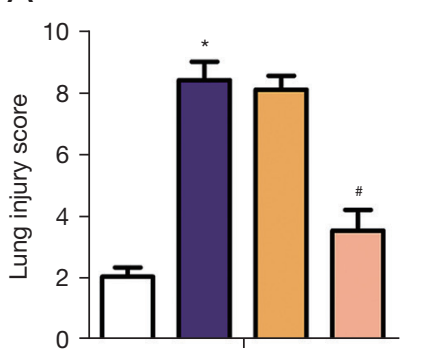

C

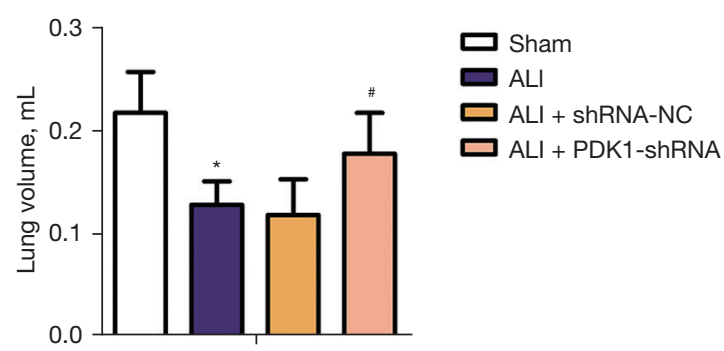

B

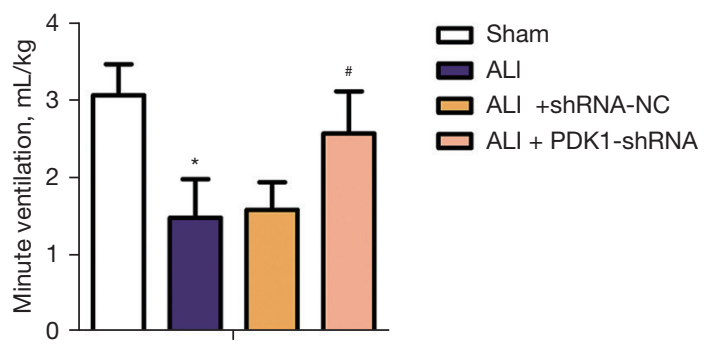

$\mathrm{D}$

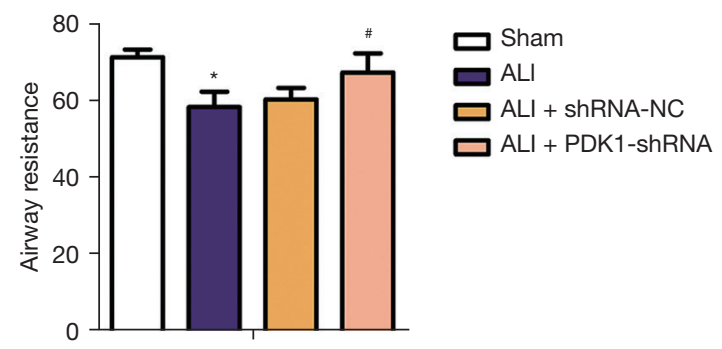

Figure 2 Effect of PDK1 silencing on lung function of rats in each group. (A) Lung injury score; (B) minute ventilation (mL/kg); (C) lung volume (mL); (D) airway resistance. *, $\mathrm{P}<0.05$ vs. the sham group; ${ }^{*}, \mathrm{P}<0.05$ vs. ALI model group. PDK1, 3-phosphoinositide-dependent kinase 1; LPS, lipopolysaccharide; mRNA, messenger RNA; ALI, acute lung injury.

main component of neutrophil cytoplasmic granules, and MPO activity in lung tissue is evaluated as an indicator of lung neutrophil accumulation (22). As shown in Figure 3B, compared with the sham group, the activity of MPO in lung tissue of the model group was significantly increased. Compared with the model group, PDK1 knockdown significantly reduced the activity of MPO in lung tissue of the rats. In order to reveal the effect of PDK1 knockdown on the inflammatory response of LPS-induced ALI model, the levels of pro-inflammatory factors IL-6, iNOS, TNF- $\alpha$, and MCP-1 were detected by ELISA. Compared with the sham group, the levels of IL- 6 , iNOS, TNF- $\alpha$, and MCP-1, IL-4 and IL-10 were significantly enhanced in the blood of LPS-induced ALI model group. In the ALI + PDK1-shRNA group, knockdown of PDK1 reversed these abnormal phenomena (Figure $3 \mathrm{C}-3 \mathrm{H}$ ). These data suggested that knockdown of PDK1 exerted and anti-inflammatory effect on LPS-induced inflammatory response.

\section{Antifibrosis effects of PDK1 knockdown in LPS-induced ALI model}

In order to reveal the effect of PDK1 knockdown on fibrosis of LPS-induced ALI model, we performed Masson staining and detected the expression of tissue fibrosis-related index proteins. The results of Masson staining revealed severe collagen deposition and fibrotic lesions in the LPS-induced model group compared with the sham group; however, PDK1 silence reduced these damages (Figure 4A). The protein level of $\alpha$-SMA and TGF- $\beta$ were detected by western blot analysis. As shown in Figure $4 B$, the protein level of $\alpha$-SMA and TGF- $\beta$ increased markedly in the LPS-induced ALI model group, while PDK1 knockdown decreased their protein levels. Hence, we inferred that knockdown of PDK1 improved lung tissue fibrosis.

\section{Effects of PDK1 knockdown on NF- $\mathrm{kB} / \mathrm{p} 65$ phosphorylation in LPS-induced ALI model}

In order to further explore the effect of PDK1 knockdown on ALI model rats, relative protein levels of NF- $\kappa B$ p-p65 was detected. According to the results of WB assay, compared with the sham group, TLR4, MyD88, and p-p65 were activated in ALI group, while PDK1 knockdown reversed these effects (Figure $5 A$ ). These results suggested that PDK1 knockdown could inhibit the TLR4/MyD88/

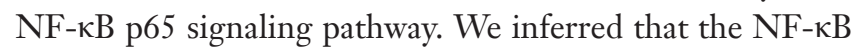
p65 signaling pathway was involved in LPS-induced proinflammatory cytokine levels and pulmonary fibrosis in the ALI model. To make the results more credible, we 
A

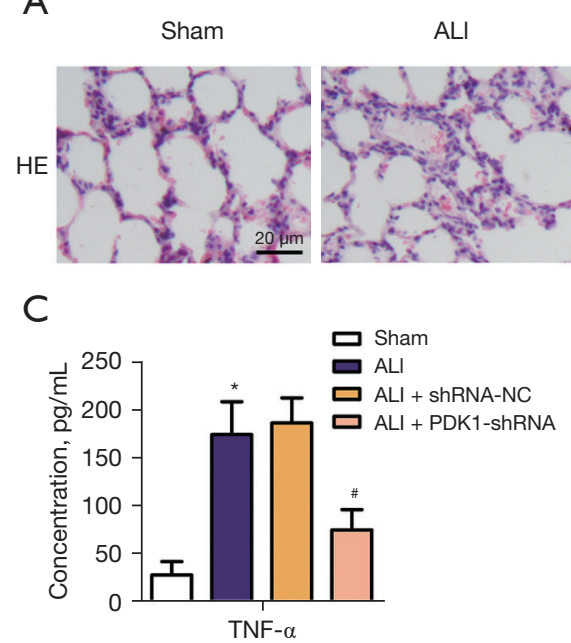

$\mathrm{F}$

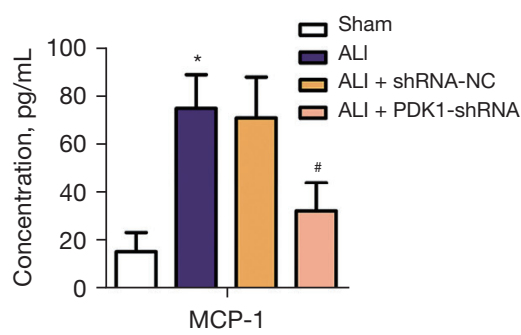

ALI + shRNA-NC $\quad$ ALI + PDK1-shRNA

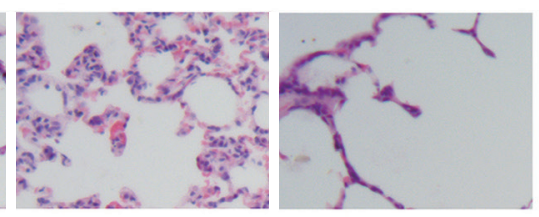

$\mathrm{D}$

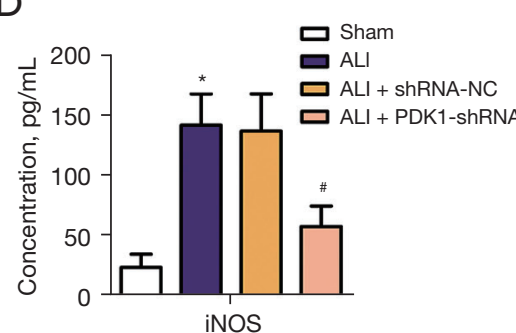

G

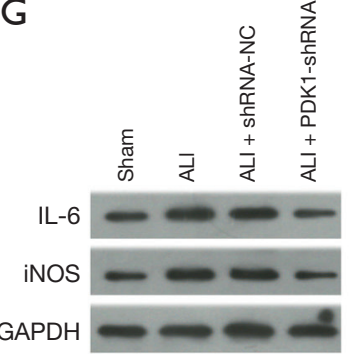

B

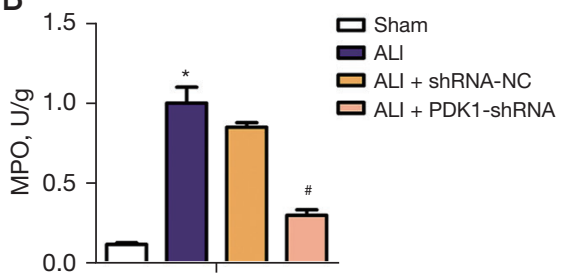

$\mathrm{E}$

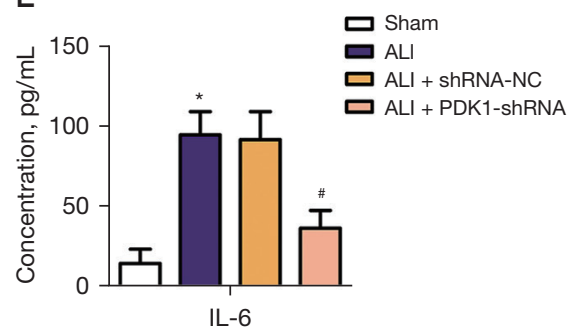

$\mathrm{H}$

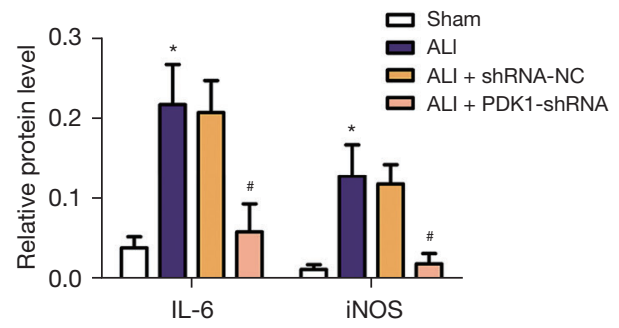

Figure 3 Anti-inflammatory effects of PDK1 silencing in LPS-induced ALI model. (A) Representative pictures of H\&E staining $(\times 200)$; (B) the content of MPO in four groups; (C-F) the concentrations of TNF- $\alpha$, iNOS, IL-6, MCP-1 in 4 groups; (G,H) relative protein level of IL-6 and iNOS. *, $\mathrm{P}<0.05$ vs. the sham group; ", $\mathrm{P}<0.05$ vs. ALI model group. PDK1, 3-phosphoinositide-dependent kinase 1; LPS, lipopolysaccharide; mRNA, messenger RNA; ALI, acute lung injury; MPO, myeloperoxidase; TNF- $\alpha$, tumor necrosis factor- $\alpha$; iNOS, inducible nitric oxide synthase; IL-6, interleukin-6; MCP-1, monocyte chemoattractant protein-1.

conducted rescue experiments using asatone, the activator of NF-кB p65. As shown in Figure 5B, compared with the control group, asatone promoted phosphorylation of p65, while PDK1 knockdown could partially offset the effect of asatone and inhibit the phosphorylation of p65. Consistently, the addition of asatone aggravated lung function damage (Figure 5C,5D), promoted lung fibrosis (Figure $5 E, 5 F$ ), and increased lung inflammation (Figure 5G,5H). However, PDK1 knockdown could partially alleviate these negative effects (Figure $5 \mathrm{C}-5 \mathrm{H}$ ). These results suggested that PDK1 knockdown attenuates LPS-induced proinflammatory cytokine levels and pulmonary fibrosis by inhibiting the NF- $\kappa \mathrm{B}$ p65 signaling pathway.

\section{Discussion}

Despite its common occurrence, ALI is a complicated inflammatory lung disease (23). Over the past decade, some progress has been made and several therapeutic strategies have been developed. Nevertheless, the mortality is still very high, reaching up to a third of cases, and a number of patients have complications due to longterm hypoxia, such as brain injury. In this study, we have discussed the effects of PDK1 interference on the levels of fibrosis and proinflammatory factors in ALI and the relationship between PDK1 interference and NF- $\mathrm{BB}$ / p56 in ALI. Our finding were as follows: (I) in vivo PDK1 interference partially reduced severe damage indexes and alleviated fibrosis in ALI; (II) PDK1 reduced high levels of proinflammatory factors, such as IL-6, iNOS, TNF- $\alpha$, and MCP-1; and (III) PDK1 interference suppressed NF-кB/ p65 pathway activation.

As a kind of regulatory protein kinase, PDK1 has been shown to be involved in the development of a variety of 
A
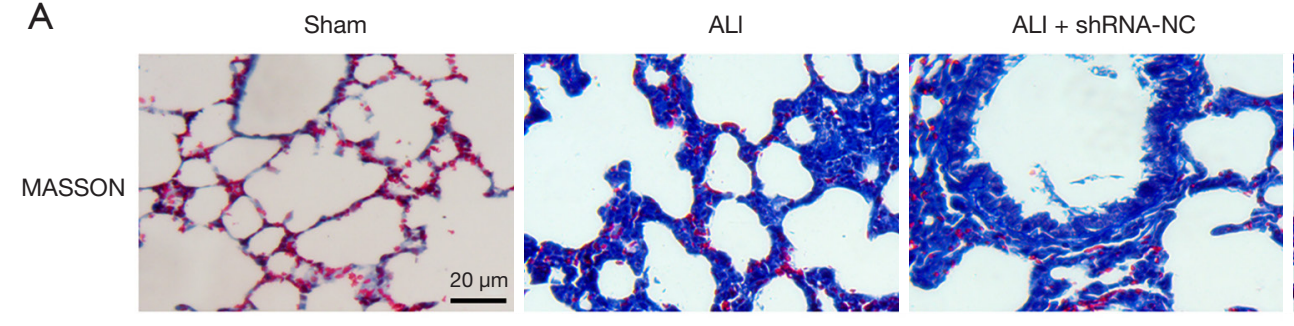

ALI + PDK1-shRNA

B
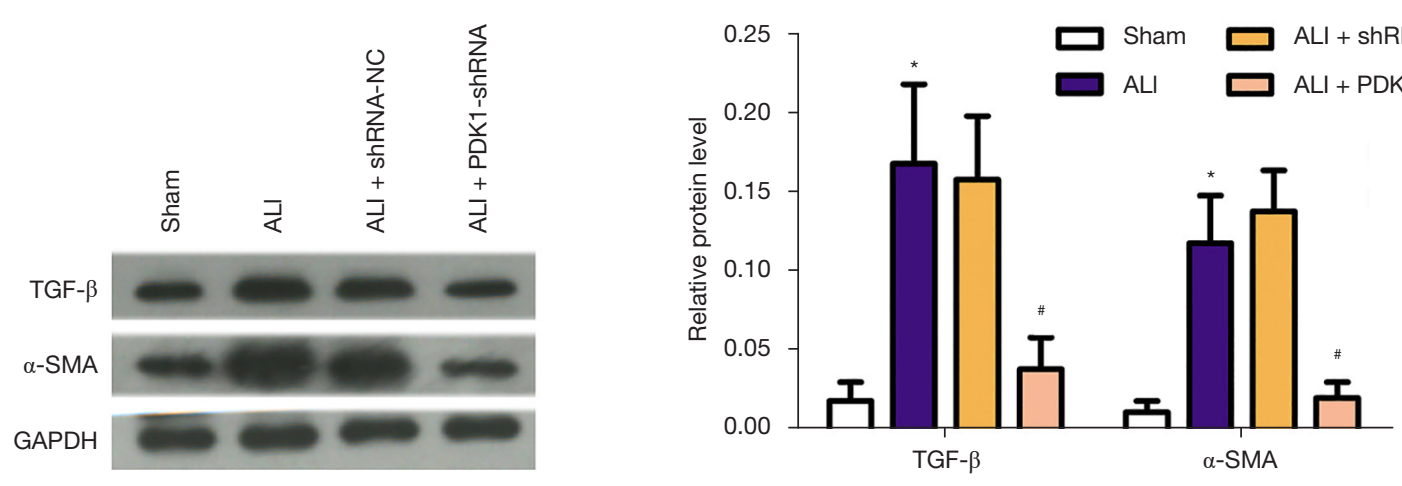

Figure 4 Anti-fibrosis effects of PDK1 silence in LPS-induced ALI model. (A) Representative pictures of Masson staining ( $\times 200$ ); (B) typical pictures of western blot assay and relative protein level of $\alpha$-SMA and TGF- $\beta$ in 4 groups. *, $\mathrm{P}<0.05$ vs. the sham group; ${ }^{*}, \mathrm{P}<0.05$ vs. ALI model group. PDK1, 3-phosphoinositide-dependent kinase 1; LPS, lipopolysaccharide; ALI, acute lung injury; TGF- $\beta$, transforming growth factor- $\beta ; \alpha$-SMA, $\alpha$-smooth muscle actin.

diseases $(14,15,24)$. Maurer et al. reported that PDK1 is overexpressed in abundant breast neoplastic lesions (24). Tao et al. found that PDK1 was involved in the process of ALI (25). It is well known that the rat model of ALI induced by LPS is considered a better ALI model. In our study, the expression levels of PDK1, whether mRNA level or protein level, were all enhanced by LPS treatment. Similarly, in our study, we found that PDK1 was overexpressed in ALI model rats. Minute ventilation and lung injury score are important indexes in the process of assessing lung injury $(26,27)$. In our study, lung injury score was enhanced, otherwise, minute ventilation, airway resistance, and lung volume were decreased in ALI model rats. We also found that the pathological damage and fibrosis of lung tissue were aggravated in ALI model rats. Fortunately, PDK1 interference not only reduced lung injury score, but also enhanced minute ventilation, airway resistance, and lung volume. Additionally, we found that PDK1 interference alleviated pathological damage and fibrosis of lung tissue. The researchers found that ALI can simultaneously induce edema formation, alveolar capillary membrane damage, and alveolar capillary membrane repair, accompanied by varying degrees of fibrosis (28-31). It is worth noting that several cytokines were shown to participate in these processes and play key roles, including TGF- $\beta 1$ and $\alpha$-SMA induced by TGF- $\beta 1(32,33)$.

The cytokine TGF- $\beta$ is extensively involved in the development of fibrosis in different organs and its role in the development of ALI had been previously reported (34). In our article, the data indicated that TGF- $\beta$ and $\alpha$-SMA were overexpressed in lung tissues of ALI rats, whereas PDK1 interference decreased the levels of $\alpha$-SMA and TGF- $\beta$. Pulmonary fibrosis is an irreversible lung disease (35), and abnormally activated myofibroblasts are the major pathological factors in progressive pulmonary fibrosis, promoting the initiation and progression of fibrosis (36). It has been demonstrated that myofibroblasts recruit inflammatory cells through the secretion of various cytokines, leading to the aggravation of inflammation $(35,37)$. HIF- $1 \alpha$ play a key role in the progression of fibrosis in various organs, including the lung (38). It has been reported that PDK-1 is a direct target gene of HIF-1 $\alpha$, and that PDK-1 induction is a critical metabolic switch adapted to cellular hypoxia by increasing glycolysis and inhibition 


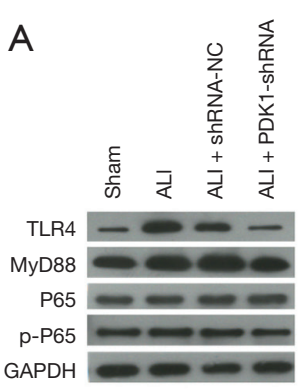

C

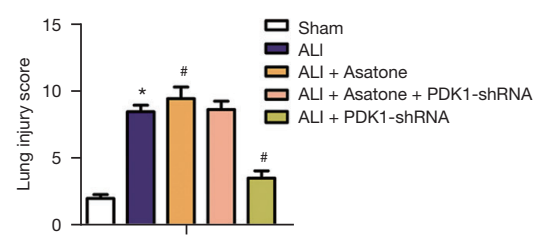

$\mathrm{F}$

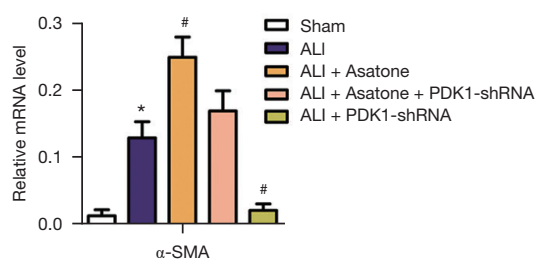

B
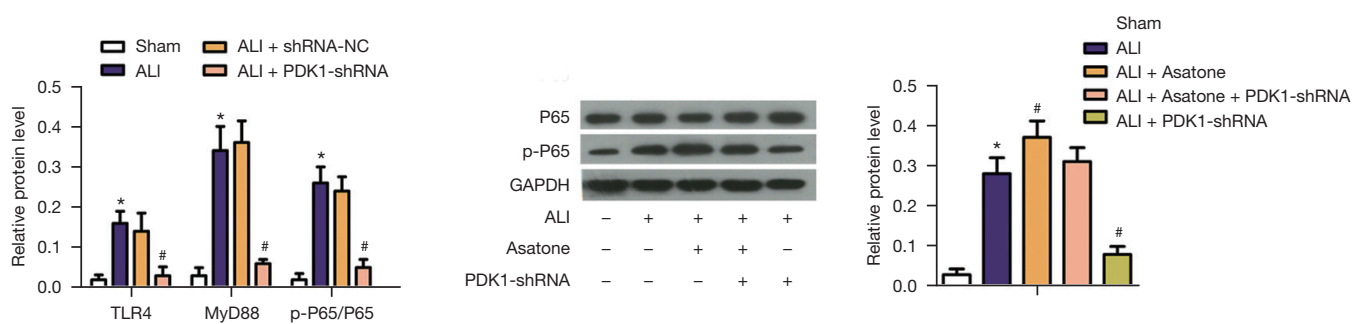

D
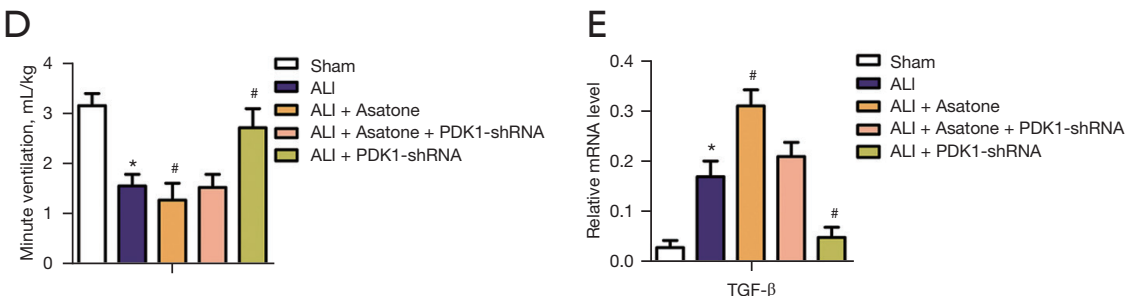

G

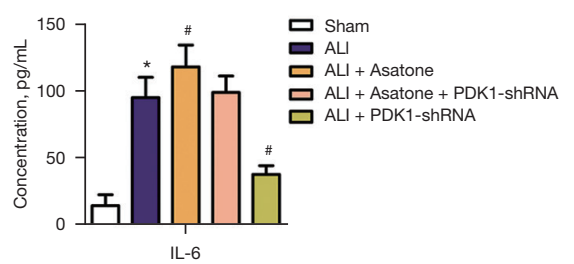

Figure 5 Effects of PDK1 silence on NF-kB p65 phosphorylation in LPS-induced ALI model. (A) Representative pictures of western blot assay and relative protein levels of TLR4, MyD88 and p-p65/p65; (B) representative pictures of western blot for the effect of asatone addition on p65 phosphorylation and relative protein level of p-p65/p65 in each group; (C) lung injury score; (D) minute ventilation (mL/kg); (E) Relative mRNA level of TGF- $\beta$; (F) relative mRNA level of $\alpha$-SMA; (G) the concentrations of IL-6; (H) the concentrations of iNOS. *, $\mathrm{P}<0.05$ vs. the sham group; ${ }^{*}, \mathrm{P}<0.05$ vs. ALI model group. PDK1, 3-phosphoinositide-dependent kinase 1; LPS, lipopolysaccharide; ALI, acute lung injury; $\alpha$-SMA, $\alpha$-smooth muscle actin; IL-6, interleukin-6; iNOS, inducible nitric oxide synthase; p-p65, phosphorylated p65.

of mitochondrial respiration (39). In the process of tissue injury and inflammation, the accumulation of hypoxia regions leads to HIF-1-mediated anaerobic glycolysis, and further provides the necessary metabolic reprogramming for the survival of hypoxic cells. TGF- $\beta$ induces the phosphorylation of the SMAD protein, thereby stimulating myofibroblast differentiation and transcription factor activation $(40,41)$. Goodwin et al. reported that hypoxia can significantly enhance TGF- $\beta$-induced myofibroblast differentiation through HIF- $1 \alpha$ and overexpression of PDK1 can sensitize glycolysis and enhance myofibroblast differentiation, and pharmacological inhibition of PDK1 effectively attenuated bleomycin-induced pulmonary fibrosis (42). Combined with previous studies, these findings suggested that knockdown of PDK1 protects fibrosis in LPS-induced ALI via regulating TGF- $\beta$.
Nguyen et al. reported that miRNA-193a targets TGF- $\beta-2$ directly and further attenuates the production of inflammatory factors IL-6, TNF- $\alpha$, and IL- $1 \beta$ induced by LPS through ERK activation (43). In the present study, the content of proinflammatory factors IL-6, MCP-1, TNF- $\alpha$, and iNOS were remarkably enhanced in LPS-induced ALI model rats; however, they were all decreased in the ALI + PDK1-shRNA group. It is well known that TLR4, MyD88, and NF- $\mathrm{KB}$ are common signal pathways regulating inflammation. Zhang et al. found that resveratrol can inhibit the production of inflammatory cytokines by regulating the TLR4, MyD88, and NF-кB signaling pathways (44). According to their report, mycetia cauliflora methanol extract targeted PDK1 to downregulate the expression of iNOS, inhibit the nuclear translocation of p65 and the phosphorylation of IкB $\alpha$, IKK, and AKT in mice with LPS- 
induced peritonitis (45). In this article, the expression levels of MyD88, TLR4, and p-p65 were significantly enhanced in LPS-induced ALI model rats, whereas, knockdown of PDK1 significantly reversed these abnormal phenomena. These results indicated that knockdown of PDK1 alleviated LPSinduced ALI via suppressing NF- $\kappa \mathrm{B} / \mathrm{p} 65$ pathway activation. To fortify conclusion, we added rescue experiments involving the addition of asatone, the activator of NF- $\kappa \mathrm{B}$ p65. As expected, the addition of asatone promoted the phosphorylation of p65, aggravated lung function damage, promoted lung fibrosis, and increased lung inflammation. However, the knockdown of PDK1 partially offset the effects of asatone. Therefore, we speculated that PDK1 knockdown inhibits fibrosis and inflammation in LPS-induced ALI

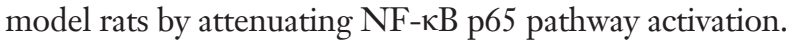

\section{Conclusions}

Our study revealed that PDK1 interference attenuated lung function injury, pathological damage, pulmonary fibrosis, and proinflammatory factors levels via regulating the NF- $\kappa \mathrm{B} / \mathrm{p} 65$ signaling pathway. However, this study was conducted on a rat model with limited sample size and clinical significance, and the underlying mechanism of lung protection of PDK1 interference requires confirmation via further research.

\section{Acknowledgments}

Funding: This research was supported by the Scientific Research Fund Project of Heilongjiang University of Traditional Chinese Medicine (201839).

\section{Footnote}

Reporting Checklist: The authors have completed the ARRIVE reporting checklist. Available at https://dx.doi. org/10.21037/atm-21-5476

Data Sharing Statement: Available at https://dx.doi. org/10.21037/atm-21-5476

Conflicts of Interest: All authors have completed the ICMJE uniform disclosure form (available at https://dx.doi. org/10.21037/atm-21-5476). The authors have no conflicts of interest to declare.

Ethical Statement: The authors are accountable for all aspects of the work in ensuring that questions related to the accuracy or integrity of any part of the work are appropriately investigated and resolved. Animal experiments were conducted according to the declaration of the Guide for the Care and Use of Laboratory Animals (2011). This study was approved by the ethics committee of Heilongjiang University of traditional Chinese Medicine (No. 2020136).

Open Access Statement: This is an Open Access article distributed in accordance with the Creative Commons Attribution-NonCommercial-NoDerivs 4.0 International License (CC BY-NC-ND 4.0), which permits the noncommercial replication and distribution of the article with the strict proviso that no changes or edits are made and the original work is properly cited (including links to both the formal publication through the relevant DOI and the license). See: https://creativecommons.org/licenses/by-nc-nd/4.0/.

\section{References}

1. Mendez JL, Hubmayr RD. New insights into the pathology of acute respiratory failure. Curr Opin Crit Care 2005;11:29-36.

2. Zhong $\mathrm{X}, \mathrm{Wu} \mathrm{Q}$, Yang $\mathrm{H}$, et al. Airway pressure release ventilation versus low tidal vol-ume ventilation for patients with acute respiratory distress syndrome/acute lung injury: a meta-analysis of randomized clinical trials. Ann Transl Med 2020;8:1641.

3. Wheeler AP, Bernard GR. Acute lung injury and the acute respiratory distress syndrome: a clinical review. Lancet 2007;369:1553-64.

4. Bellani G, Laffey JG, Pham T, et al. Epidemiology, Patterns of Care, and Mortality for Patients With Acute Respiratory Distress Syndrome in Intensive Care Units in 50 Countries. JAMA 2016;315:788-800.

5. Stapleton RD, Wang BM, Hudson LD, et al. Causes and timing of death in patients with ARDS. Chest 2005;128:525-32.

6. Singh N. Acute lung injury and acute respiratory distress syndrome. Lancet 2007;370:383-4; author reply 384-5.

7. Chen $\mathrm{H}$, Bai C, Wang $\mathrm{X}$. The value of the lipopolysaccharide-induced acute lung injury model in respiratory medicine. Expert Rev Respir Med 2010;4:773-83.

8. Sato K, Kadiiska MB, Ghio AJ, et al. In vivo lipid-derived free radical formation by NADPH oxidase in acute lung injury induced by lipopolysaccharide: a model for ARDS. FASEB J 2002;16:1713-20.

9. Kim CO, Huh AJ, Han SH, et al. Analysis of cellular 
senescence induced by lipopolysaccharide in pulmonary alveolar epithelial cells. Arch Gerontol Geriatr 2012;54:e35-41.

10. Yoshida T, Nagai K, Inomata T, et al. Relationship between neutrophil influx and oxidative stress in alveolar space in lipopolysaccharide-induced lung injury. Respir Physiol Neurobiol 2014;191:75-83.

11. Sheu CC, Gong MN, Zhai R, et al. Clinical characteristics and outcomes of sepsis-related vs non-sepsis-related ARDS. Chest 2010;138:559-67.

12. Kong G, Huang X, Wang L, et al. Astilbin alleviates LPSinduced ARDS by suppressing MAPK signaling pathway and protecting pulmonary endothelial glycocalyx. Int Immunopharmacol 2016;36:51-8.

13. Xiao Q, Zheng F, Wu J, et al. Activation of ERK and Mutual Regulation of Stat3 and SP1 Contribute to Inhibition of PDK1 Expression by Atractylenolide-1 in Human Lung Cancer Cells. Cell Physiol Biochem 2017;43:2353-66.

14. Gagliardi PA, di Blasio L, Primo L. PDK1: A signaling hub for cell migration and tumor invasion. Biochim Biophys Acta 2015;1856:178-88.

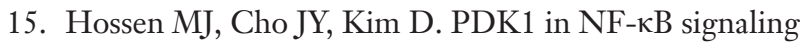
is a target of Xanthium strumarium methanolic extractmediated anti-inflammatory activities. J Ethnopharmacol 2016;190:251-60.

16. Luo Q, Yan X, Bobrovskaya L, et al. Antineuroinflammatory effects of grossamide from hemp seed via suppression of TLR-4-mediated NF- $\mathrm{NB}$ signaling pathways in lipopolysaccharide-stimulated BV2 microglia cells. Mol Cell Biochem 2017;428:129-37.

17. Wang J, Liu Y'T, Xiao L, et al. Anti-inflammatory effects of apigenin in lipopolysaccharide-induced inflammatory in acute lung injury by suppressing COX-2 and NF-kB pathway. Inflammation 2014;37:2085-90.

18. Kang JL, Lee HW, Lee HS, et al. Genistein prevents nuclear factor-kappa B activation and acute lung injury induced by lipopolysaccharide. Am J Respir Crit Care Med 2001;164:2206-12.

19. Zhang ZB, Xu QP. Experimental Study of Ginsenoside Rg1 Combined with Antibiotics in the Treatment of Acute Lung Injury in Mice with Sepsis. Sichuan Da Xue Xue Bao Yi Xue Ban 2020;51:371-5.

20. Hagiwara S, Iwasaka H, Matumoto S, et al. Effects of an angiotensin-converting enzyme inhibitor on the inflammatory response in in vivo and in vitro models. Crit Care Med 2009;37:626-33.

21. Murakami K, McGuire R, Cox RA, et al. Heparin nebulization attenuates acute lung injury in sepsis following smoke inhalation in sheep. Shock 2002;18:236-41.

22. Kingir ZB, Özdemir Kural ZN, Cam ME, et al. Effects of dapagliflozin in experimental sepsis model in rats. Ulus Travma Acil Cerrahi Derg 2019;25:213-21.

23. Xu WM, Zhang JY, Liu J. Effects of emulsified isoflurane preconditioning on LPS-induced acute lung injury in rats. Sichuan Da Xue Xue Bao Yi Xue Ban 2013;44:554-7.

24. Maurer M, Su T, Saal LH, et al. 3-Phosphoinositidedependent kinase 1 potentiates upstream lesions on the phosphatidylinositol 3-kinase pathway in breast carcinoma. Cancer Res 2009;69:6299-306.

25. Tao J, Nie Y, Hou Y, et al. Chemomics-Integrated Proteomics Analysis of Jie-Geng-Tang to Ameliorate Lipopolysaccharide-Induced Acute Lung Injury in Mice. Evid Based Complement Alternat Med 2016;2016:7379146.

26. Xu Z, Gu L, Bian Q, et al. Oxygenation, inflammatory response and lung injury during one lung ventilation in rabbits using inspired oxygen fraction of 0.6 vs. 1.0. J Biomed Res 2016;31:56-64.

27. Zhu R, Zhao Y, Li X, et al. Effects of penehyclidine hydrochloride on severe acute pancreatitis-associated acute lung injury in rats. Biomed Pharmacother 2018;97:1689-93.

28. Dhami R, He X, Schuchman EH. Acid sphingomyelinase deficiency attenuates bleomycin-induced lung inflammation and fibrosis in mice. Cell Physiol Biochem 2010;26:749-60.

29. Waerntges S, Klingel K, Weigert C, et al. Excessive transcription of the human serum and glucocorticoid dependent kinase hSGK1 in lung fibrosis. Cell Physiol Biochem 2002;12:135-42.

30. Siegmann N, Worbs D, Effinger F, et al. Invariant natural killer T (iNKT) cells prevent autoimmunity, but induce pulmonary inflammation in cystic fibrosis. Cell Physiol Biochem 2014;34:56-70.

31. Grassmé H, Carpinteiro A, Edwards MJ, et al. Regulation of the inflammasome by ceramide in cystic fibrosis lungs. Cell Physiol Biochem 2014;34:45-55.

32. Rodríguez A, Castaño M, Peña L, et al. Immunocytochemical detection of growth factors (PDGF and TGF beta) in equine chronic pneumonia. Res Vet Sci 1996;60:82-7.

33. Bonner JC, Brody AR. Asbestos-induced alveolar injury. Evidence for macrophage-derived PDGF as a mediator of the fibrogenic response. Chest 1991;99:54S-5S.

34. Alghetaa H, Mohammed A, Sultan M, et al. Resveratrol protects mice against SEB-induced acute lung injury and 
mortality by miR-193a modulation that targets TGF- $\beta$ signalling. J Cell Mol Med 2018;22:2644-55.

35. Wynn TA. Integrating mechanisms of pulmonary fibrosis. J Exp Med 2011;208:1339-50.

36. Todd NW, Luzina IG, Atamas SP. Molecular and cellular mechanisms of pulmonary fibrosis. Fibrogenesis Tissue Repair 2012;5:11.

37. Wilson MS, Wynn TA. Pulmonary fibrosis: pathogenesis, etiology and regulation. Mucosal Immunol 2009;2:103-21.

38. Ueno M, Maeno T, Nomura M, et al. Hypoxia-inducible factor- $1 \alpha$ mediates TGF- $\beta$-induced PAI- 1 production in alveolar macrophages in pulmonary fibrosis. Am J Physiol Lung Cell Mol Physiol 2011;300:L740-52.

39. Papandreou I, Cairns RA, Fontana L, et al. HIF-1 mediates adaptation to hypoxia by actively downregulating mitochondrial oxygen consumption. Cell Metab 2006;3:187-97.

40. Frangogiannis $N$. Transforming growth factor- $\beta$ in tissue fibrosis. J Exp Med 2020;217:e20190103.

41. Saito A, Horie M, Nagase T. TGF- $\beta$ Signaling in Lung Health and Disease. Int J Mol Sci 2018;19:2460.

Cite this article as: Yang $\mathrm{K}, \mathrm{Li} \mathrm{B}$, Chen J. Knockdown of phosphoinositide-dependent kinase 1 (PDK1) inhibits fibrosis and inflammation in lipopolysaccharide-induced acute lung

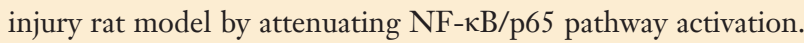
Ann Transl Med 2021;9(22):1671. doi: 10.21037/atm-21-5476
42. Goodwin J, Choi H, Hsieh MH, et al. Targeting HypoxiaInducible Factor-1 $\alpha /$ Pyruvate Dehydrogenase Kinase 1 Axis by Dichloroacetate Suppresses Bleomycininduced Pulmonary Fibrosis. Am J Respir Cell Mol Biol 2018;58:216-31.

43. Nguyen DN, Sangild PT, Ostergaard MV, et al. Transforming growth factor- $\beta 2$ and endotoxin interact to regulate homeostasis via interleukin-8 levels in the immature intestine. Am J Physiol Gastrointest Liver Physiol 2014;307:G689-99.

44. Zhang Z, Chen N, Liu JB, et al. Protective effect of resveratrol against acute lung injury induced by lipopolysaccharide via inhibiting the myd88-dependent Toll-like receptor 4 signaling pathway. Mol Med Rep 2014;10:101-6.

45. Jeong SG, Kim S, Kim HG, et al. Mycetia cauliflora methanol extract exerts anti-inflammatory activity by directly targeting PDK1 in the NF- $\kappa \mathrm{B}$ pathway. $\mathrm{J}$ Ethnopharmacol 2019;231:1-9.

(English Language Editor: J. Jones) 